\title{
10.7 TEMPERATURE AND WIND VELOCITY OSCILLATIONS ALONG A GENTLE SLOPE DURING SEA-BREEZE EVENTS.
}

\author{
Sophie Bastin ${ }^{(*)}$, Philippe Drobinski \\ IPSL, Paris, France;
}

\section{$1^{*}$. INTRODUCTION}

The flow structure at Vallon d'Ol (43.35 N, $\left.5.4^{\mathrm{C}} \mathrm{E}\right)$ on a gentle slope in the northern suburbs of Marseille in southern France has been documented by means of surface wind and temperature measurements collected from 7 June to 14 July 2001 during the ESCOMPTE experiment ('Field experiment to constrain models of atmospheric pollution and emissions transport') (Cros et al., 2004). The analysis of the time series reveals temperature and wind speed oscillations during several nights and several days during the whole observing period.

Oscillating katabatic winds have been reported in the literature from theoretical, experimental and numerical studies. In the present study, the dynamics of the observed oscillating katabatic winds are in good agreement with the theory. Comparatively to katabatic winds, no daytime observations of oscillating anabatic upslope flows have ever been published, probably because of temperature inversion breakup that inhibits upslope winds. The present paper shows that cold air advection by sea-breeze generates a mesoscale horizontal temperature gradient that forces the baroclinicity of the atmosphere which then allows low-frequency oscillations, similarly to katabatic flow. An expression of the oscillation period is derived which accounts for the contribution of the sea-breeze induced mesoscale horizontal temperature gradient. The theoretical predictions of the oscillation period are compared to the measurements and a very good agreement is found.

\section{OBSERVATIONS}

Vallon d'Ol is an isolated hill located in the north of Marseille. The instrumental site is at $270 \mathrm{~m}$ above sea level (ASL) and it is close to the coastline (Fig. 1), which makes this site strongly influenced by the thermal induced breeze circulation that often develops in this region during summertime (Bastin et al., 2004).

* Corresponding author address: Sophie Bastin, Service d'Aéronomie, Université Pierre et Marie Curie, Tour 45, 4 Place Jussieu, 75252 Paris Cedex 05, France.

e-mail: sophie.bastin@aero.jussieu.fr
The direction of the sea-breeze that blows over Vallon d'Ol is nearly $240^{\circ}$ since the coastline has a northwest-southeast orientation. The following data are used in this study (Fig.1):

- at Vallon d'Ol (VDO) (sloping terrain) (43.35 N, 5.4 E): wind speed and direction at $10 \mathrm{~m}$ above ground level (AGL) and temperature and humidity at $2 \mathrm{~m}$ AGL with a time resolution of $5 \mathrm{~min}$; vertical profiles of the wind speed and direction, and the virtual potential temperature between $50 \mathrm{~m}$ up to $400 \mathrm{~m}$ AGL (at most) with a $25 \mathrm{~m}$ increment.

- at Barden (flat terrain) (BAR) (43.629N, 5.20E): same as Vallon d'Ol for surface measurements.

- at Marseille Observatory (OBS) (43.39N, 5.4 $\mathrm{E}$ ): radiative fluxes

- at Aix en Provence (AIX) (43.5 N, 5.4的) : radiosondes

- at mesoscale: temperature at $2 \mathrm{~m}$ AGL by the operational meteorological surface station network. These data were used to determine the mesoscale temperature gradient that drives the sea-breeze flow in this area.
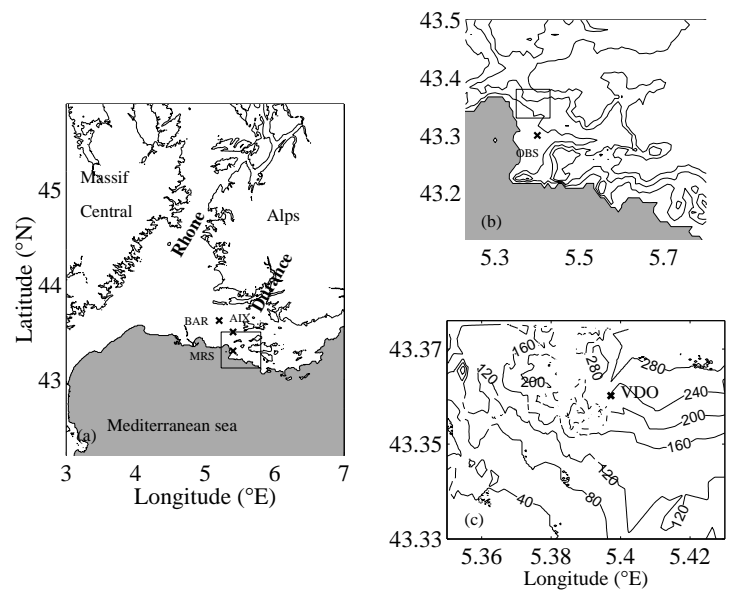

Fiqure 1: (a) Topography of the ESCOMPTE target area. Sea surfaces are shaded. The isocontour corresponds to $500 \mathrm{~m}$ ASL. BAR, AIX and MRS are the acronyms for Barden, Aix and Marseille. (b) zoom of the Marseille area. Contours interval is 100 $m$ from sea level to $300 \mathrm{~m}$ ASL. Acronym OBS indicates the location of Marseille Observatory. (c) zoom of panel $b$. The contour interval is $40 \mathrm{~m}$. Acronym VDO is for Vallon d'Ol.

\subsection{Observations on 25 June 2001}

The synoptic situation for this day is anticyclonic and sea-breeze blows over Vallon 
d'OI. During nighttime (00-08 local time (LT) (UTC+2)), the wind is very weak $\left(\approx 1 \mathrm{~m} \mathrm{~s}^{-1}\right)$ and the mean value for its direction is nearly $70^{\circ}$ (northeast) (Fig.2). The transition between nighttime and daytime is well marked. During daytime, the wind direction is about $250^{\circ}$, corresponding to the sea-breeze and maximum slope directions at Vallon d'Ol (Fig.1c) and the wind speed increases from 1 to $4 \mathrm{~m} \mathrm{~s}^{-1}$. The observed wind is likely to be a combination of the two thermally driven winds and the influence of the local perturbation due to the sea-breeze on the upslope flow cannot be assessed from the data.

Figure 2 and $3 \mathrm{c}$ clearly shows the existence of temperature and velocity oscillations. These oscillations have a larger magnitude during daytime than during nighttime. Figure 3 shows the nighttime and daytime temperature oscillations as a function of time (panels a and c) and the corresponding power spectrum (panels $b$ and d). It reveals an oscillation period of about $1 \mathrm{hr}$ with a $1.5 \mathrm{hr}$ modulation during nighttime, similarly to Helmis and Papadopoulos (1996) who showed evidence of the co-existence of several time scales (which they attribute to the combination of the katabatic downslope flow with synoptic flow). The north-east component of the oscillating katabatic flow is consistent with Hérnandez et al. (1998) who showed from numerical simulations, that the air drains off the mountain along the maximum slope direction.
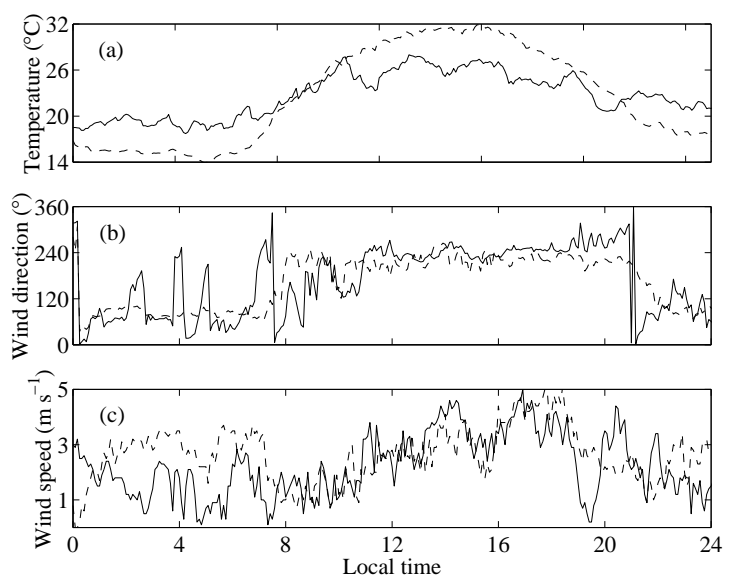

Figure 2: Time evolution of surface temperature (a), wind direction (b) and speed (c) on 25 June 2001 between 0000 and 2400 LT at Vallon d'Ol (solid line) and Barden (dashed line) (see Fig.1)

During daytime, the temperature oscillates between $24^{\circ} \mathrm{C}$ and $28^{\circ} \mathrm{C}$ with a period of nearly $3 \mathrm{hrs}$ (Fig.3d). Looking in detail, the period is not strictly constant. The time period increases from about $150 \mathrm{~min}$ at the beginning of the day up to $210 \mathrm{~min}$ in the evening. The wind speed displays periodic intensifications too, with the same period but with a phase shift with respect to the temperature time series. Figure 2 also shows that the sea-breeze flow reaches Barden at 08 $\mathrm{LT}$ and that the wind reverses at $22 \mathrm{LT}$, but no temperature or wind speed oscillation is visible at Barden. This corroborates the fact that temperature and wind oscillation at Vallon d'OI results from the interaction between the seabreeze flow and the slope since the terrain is flat in the Barden area. The time series of virtual potential temperature at different heights above ground level on 25 June 2001 (not shown), as retrieved from the RASS sodar located at Vallon d'OI indicate that the oscillations exist within a 250 m deep layer which is embedded in the sea-breeze circulation of about 1000 m depth (Bastin et al.,2004).
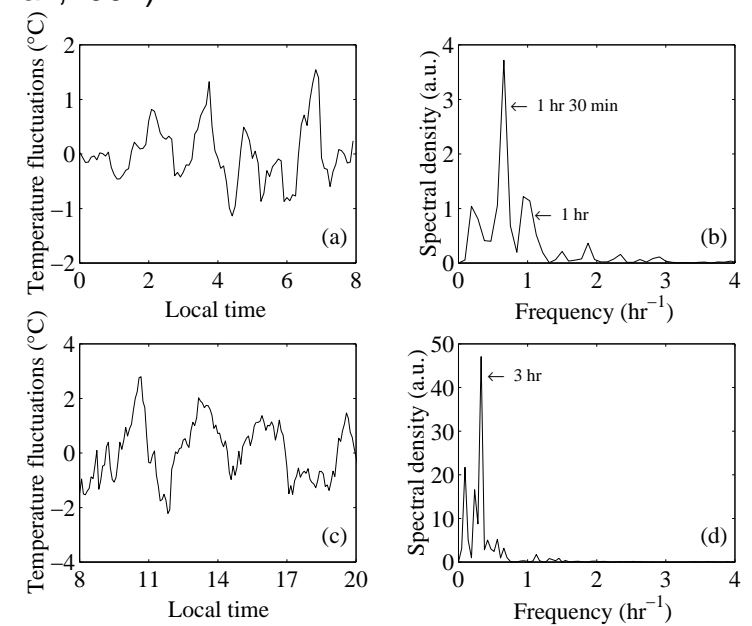

Figure 3: Time series of the temperature fluctuations on 25 June 2001 at Vallon d'Ol between 0000 and 0800 LT (a) with its Fourier transform (b). Panels $\sim c$ and $d$ are similar to panels a and $b$ for the time series between 0800 and 2000 LT.

\subsection{Statistical analysis}

During the ESCOMPTE campaign, several nights and days have presented this type of periodical fluctuations in the Marseille area, in particular at Vallon d'OI. Table I indicate the days when the surface measurements at Vallon d'Ol show evidence of temperature and velocity oscillations during the ESCOMPTE experiment. It shows that the wind direction has a west-southwest component for all these days. This point is confirmed when looking the distributions of wind speed and direction for daytime periods (not shown). The wind direction distribution for the whole observing period shows three main flow patterns (southeast, southwest and northwest). When oscillations occur, only the southwest flow pattern remains, corresponding to the sea-breeze and maximum slope 
directions at Vallon d'OI. Table I shows that the daytime oscillation period varies between 100 and $180 \mathrm{~min}$, while this period ranges from 45 to 90 during nighttime. The depth of the layer at Vallon d'Ol where temperature and wind speed oscillations are visible is inferred from the RASS sodar measurements and ranges from 100 to $300 \mathrm{~m}$.

\begin{tabular}{cccc}
\hline $\begin{array}{c}\text { Date } \\
\text { (2001) }\end{array}$ & direction & Speed $\left(\mathrm{m} \mathrm{s}^{-1}\right)$ & $\begin{array}{c}\text { Oscillation } \\
\text { period }(\mathrm{min})\end{array}$ \\
\hline 7 June & W/SW & 6 & 100 \\
13 June & $\mathrm{SW}$ & 6 & 180 \\
20 June & $\mathrm{SW}$ & 5 & 130 \\
23 June & W/SW & 5 & 180 \\
25 June & WSW & 4 & 180 \\
26 June & W/SW & 4 & 120 \\
8 July & $\mathrm{SW}$ & 7 & 120 \\
13 July & $\mathrm{SW}$ & 5 & 150 \\
\hline Table I: Daytime periods when the surface \\
measurements at Vallon d'Ol shiw evidence of \\
temperature and velocity oscillations.
\end{tabular}

\section{DISCUSSION}

\subsection{Theoritical considerations.}

The main difference between daytime and nighttime slope flows is the creation of unstability when the air adjacent to the surface is warmed more than the free air just above it. Radiative heating creates a horizontal pressure gradient that drives the upslope flow but, in the same time, convection and turbulence are generated to reverse the vertical unstability of the atmosphere. If convection is strong, the 'buoyancy layer' may be fully eroded by mixing, temperature inversion breakup can occur and upslope winds can be inhibited (Whiteman, 1982a,b). The dynamical process we suggest here to explain the oscillating character of the upslope wind is the following: the advection of cold air by sea-breeze imposes a mesoscale temperature gradient that forces the baroclinicity of the atmosphere. This temperature gradient is maintained all day long. The mechanism of the observed oscillations is thus very similar to lowfrequency oscillations of katabatic flows: (i) the isentrope inclination allows the sea-breeze flow to run along the slope; (ii) the adiabatic cooling due to the air ascension retards the flow; (iii) the radiative heating near the surface allows the flow to accelerate.

Following Mahrt (1982) or Manins and Sawford (1979) and by writing the potential temperature as follows:

$$
\begin{aligned}
\theta & =\bar{\theta}+\theta^{*} \\
& =\theta_{0}+\Gamma z+\frac{\partial\left(\bar{\theta}+\theta^{*}\right)}{\partial x} x \\
& =\theta_{0}+\theta^{\prime}
\end{aligned}
$$

(where $\theta_{0}$ is the reference potential temperature, $\bar{\theta}(\mathrm{x}, \mathrm{z}, \mathrm{t})$ the mesoscale potential temperature field and $\theta^{*}(x, z, t)$ is the perturbation with regards to the mesoscale field due to the existence of the gravity flow. $\partial \bar{\theta} / \partial x$ is the horizontal gradient of potential temperature due to the existence of the thermally-induced sea-breeze flow (it is supposed to be spatially constant), $\partial \bar{\theta} / \partial z$ is the vertical lapse rate of the atmosphere. It is noted $\gamma . \Gamma\left(=\gamma+\partial \theta^{*} / \partial z\right)$ and the horizontal gradient of the temperature perturbation are also supposed to be spatially constant. $\theta^{\prime}$ represents the total deviation of $\theta$ from the reference value), we can obtain the following differential equation set (using approximation of hydrostatic):

$$
\left\{\begin{aligned}
\frac{\partial V}{\partial t}= & \frac{g}{\theta_{0}} \sin \alpha \Theta+F \\
& +\frac{g \cos \alpha}{\theta_{0}}\left(\Gamma \sin \alpha+\frac{\partial\left(\bar{\theta}+\theta^{*}\right)}{\partial x} \cos \alpha\right) \frac{h}{2} \\
\frac{\partial \Theta}{\partial t}= & -V\left(\frac{\partial\left(\bar{\theta}+\theta^{*}\right)}{\partial x} \cos \alpha+\Gamma \sin \alpha\right)+Q
\end{aligned}\right.
$$

where $\mathrm{V}$ is the bulk velocity along the slope, $\Theta$ the bulk potential temperature (averaged over the warmed surface layer of depth $h$ ), $F$ a general frictional force, $Q$ the warming rate averaged over $h$ and $x$ the horizontal axis oriented in the direction of the maximum slope. By combining and differentiating these two equations, we obtain a differential equation which describes a harmonic oscillation of wind with the following pulsation frequency: (1)

$$
\omega=\sqrt{\frac{g}{\theta_{0}} \sin \alpha\left(\frac{\partial\left(\bar{\theta}+\theta^{*}\right)}{\partial x} \cos \alpha+\Gamma \sin \alpha\right)}
$$

This expression is valid for both daytime and nighttime periods. It is very similar to that found in the literature except that we have additional terms here, the most important is $\frac{\partial \bar{\theta}}{\partial x} \cos \alpha$ that is associated with the sea-breeze flow. The sea breeze flow maintains baroclinicity 
and allows the atmosphere to be locally stable. The order of magnitude of $\partial \theta / \partial x$ during daytime is $0.1^{\circ} \mathrm{km}^{-1}$ and it is smaller in absolute value during nighttime when the land breeze blows. The vertical gradient of potential temperature $\gamma$ is about $10^{\circ} \mathrm{km}^{-1}$ during the night and it can be zero in the atmospheric boundary layer during daytime. During daytime, the horizontal and vertical gradient of $\theta^{*}$ are progressively cancelled due to convection. Thus, during nighttime, the main contribution to the oscillation is generally the vertical stratification and the expression of the oscillation period can be reduced to $2 \Pi /(N \sin \alpha)$, where $N$ is the Brunt-Väisälä frequency $\left(N^{2}=g / \theta_{0} \Gamma\right)$, as derived by Schumann (1990). During daytime, for small slope angle (i.e. $\cos \alpha=\mathrm{O}(1)$ and $\sin$ $\alpha=O(\alpha)$ ), the horizontal mesoscale temperature gradient can dominate over the other terms which sometimes can be negligible

\subsection{Application to the observations}

The nighttime oscillation periods measured at Vallon d'Ol are in good agreement with Schumann's theoretical prediction. For instance, on the nights 24/25 and 26/27 June 2001, $N$ retrieved from the RASS sodar data is about $0.03 \mathrm{~s}^{-1}$ which leads to an oscillation period of $67 \mathrm{~min}$, while the measured values are 60 and $50 \mathrm{~min}$ respectively. On the night $25 / 26, N \cong 0.024 \mathrm{~s}^{-1}$ so that the oscillation period is approximately $87 \mathrm{~min}$, which is in fairly good agreement with the observations (80 $\mathrm{min})$.

During daytime, both the horizontal and vertical temperature gradients impact on the oscillation period. Indeed, on 25 June 2001, the oscillation varies with time: at the beginning of the day, it is about $150 \mathrm{~min}$ and it is about $210 \mathrm{~min}$ at the end of the day. Figures 4 and 5 provide an explanation of the time evolution of this period. Figure 4 displays two averaged vertical profiles of the virtual potential temperature (equal to $\Gamma$ ) extracted from the RASS sodar data. Solid line corresponds to the morning profile averaged between 1030 and 1300 LT and the dashed line to the afternoon profile averaged between 1400 and 1600 LT. It clearly shows the development of a convective boundary layer, so the oscillation period is expected to increase as shown by Eq.1.

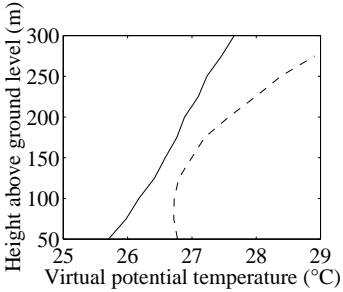

Fig.4: Averaged vertical profiles of $\theta$ in the morning (solid line) and in the afternoon (dashed line).

However, Fig.5 shows that $\partial \bar{\theta} / \partial x$ increases all day long. It is nearly $0^{\circ} \mathrm{km}^{-1}$ in the morning (10-11 LT) when $\partial \bar{\theta} / \partial z \cong 7^{\circ} \mathrm{km}^{-1}$, it is about $0.15^{\circ} \mathrm{km}^{-1}$ between 12 and $16 \mathrm{LT}$ when $\partial \bar{\theta} / \partial z \cong 0^{\circ} \mathrm{km}^{-1}$ and $\partial \bar{\theta} / \partial x$ reaches its maximum value at $18 \mathrm{LT}$ before decreasing and reversing. The impossibility to estimate the value of $\partial \theta^{*} / \partial x$ from observations is likely not to be a real problem since during daytime $\partial \theta^{*} / \partial x \cong 0$ due to convection and turbulence. From these values, Eq.1 gives an oscillation period of $132 \mathrm{~min}$ in the morning (before 12 LT), and $207 \mathrm{~min}$ in the afternoon (before 17 LT), which are good estimates of the observed oscillation period which varies between about 150 and $210 \mathrm{~min}$. Furthermore, the comparison between the observed daytime oscillation periods and the theoretical prediction which includes the contribution of the mesoscale horizontal temperature gradient was found to be in good agreement for the whole ESCOMPTE campaign. For some days, the wind is oriented in the same direction as the sea-breeze flow (southwest) but no oscillation is visible. Except for one case (24 June 2001), the absence of vertical and horizontal gradient explains the absence of oscillations which is consistent with Eq.1 (the oscillation period tends to infinity). On 24 June 2001, the reason why oscillations do not occur is unknown.

\section{CONCLUSION}

This study shows the existence of temperature and wind velocity oscillations measured during nighttime and daytime at Vallon d'Ol on a gentle slope in southern France. During nighttime, the mechanism that generates these oscillations is that proposed by Fleagle (1950) and the expression of the oscillation period: $(2 \Pi /(\mathrm{N} \sin \alpha))$ derived by Schumann (1990) gives good agreement with observed values. During daytime, the theoritical expression of the pulsation accounts for the existence of the mesoscale temperature 
gradient and theory is in most cases in good agreement with observations.

\section{REFERENCES}

Bastin, S., Drobinski, P., Dabas, A.M., Delville, P., Reitebuch, O., Werner, C.: 2004, 'Impact of the Rh/^one and Durance Valleys on Sea-Breeze Circulation in the Marseille Area', Atmos. Res., in press.

Cros B., et al.: 2004, 'The ESCOMPTE Program: An Overview', Atmos. Res., in press.

Fleagle R.G.: 1950, 'A Theory of Air Drainage', J. Meteorol., 7, 227-232.

Helmis, C.G., and Papadopoulos, K.H.: 1996, 'Some Aspects of the Variation with Time of Katabatic Flow over a Simple Slope', Quart. J. Roy. Meteorol. Soc., 122, 595610.

Hernandez, E., De Las Parras, J., Martin, I., and Rua, A.: 1998, 'A Field Case Study and Numerical Simulation of Mountain Flows with Weak Ambient Winds', J. Appl. Meteorol., 37, 623-637.

Mahrt, L.: 1982, 'Momentum balance of gravity flows', \{lit J. Atmos. Sci.\}, \{lbf 39\}, 27012711.

Manins, P.C., and Sawford, B.L.: 1979, 'A Model of Katabatic Winds', \{lit J. Atmos. Sci. $\},\{$ bf 36\}, 619-630.

Schumann, U.: 1990, 'Large-Eddy Simulation of the Up-Slope Boundary Layer', Quart. J. Roy. Meteorol. Soc., 116, 637-670.

Whiteman, C.D., and McKee, T.B.: 1982a, Breakup of Temperature Inversions in Deep Mountain Valleys: Part 1. Observations', J. Appl. Meteorol., 21, 270289.

Whiteman, C.D., and McKee, T.B., 1982b: Breakup of Temperature Inversions in Deep Mountain Valleys: Part 2. Thermodynamic Model', J. Appl. Meteorol., 21, 290-302. 